Leo T. Chylack, Jr., M.D. served NASA as principal investigator on the NASCA project since its inception in 2003. He served as Chief of Ophthalmology at the Brigham and Women's Hospital (BWH) from 1971 until 1999. He directed the BWH's Center of Clinical Cataract Research and later the Center for Ophthalmic Research for the past three decades. He served as Professor and Vice-Chairman (Research) in the Department of Ophthalmology at Harvard Medical School (HMS) from 1989 until 2009. In August 2010, he retired from the BWH and HMS to serve as President of a company he formed, Chylack Inc. 


\section{NASCA Report 2: Longitudinal Study of Relationship of Exposure to Space Radiation and Risk of Lens}

Opacity by Leo T. Chylack, Jr., M.D. ${ }^{a}$, Leif E. Peterson, Ph.D. ${ }^{b}$, Alan H. Feiveson, Ph.D. ${ }^{c}$, William H. Tung ${ }^{a}$, Mary L. Wear, Ph.D. ${ }^{d}$, Lisa J. Marak, R.N. ${ }^{d}$, Dale S. Hardy, Ph.D. ${ }^{b}$, Francis A. Cucinotta, Ph.D. ${ }^{c}$ from: a. Center for Ophthalmic Research, Brigham and Women's Hospital, Boston, MA, b. The Methodist Hospital Research Institute, Houston, TX, c. Lyndon B. Johnson Space Center, NASA, Houston, TX, d. Wyle Integrated Science and Engineering, Houston, TX, e. Space Center Eye Associates, Houston, TX.

Introduction: The NASA Study of Cataract in Astronauts (NASCA) was a five-year longitudinal study of the effect of space radiation exposure on the severity/progression of nuclear $(\mathrm{N})$, cortical (C), and posterior subcapsular (PSC) lens opacities. It began in 2003 and was completed in December, 2009. Participants included 171 consenting astronauts who flew at least one mission in space, and comparison subjects consisted of three groups, a) 53 astronauts who had not flown in space, b) 95 military aircrew personnel, and c) 99 non-aircrew, ground-based subjects.

Methods: Continuous measures of $\mathrm{N}$ (pixel density), C (\% area opaque), and PSC ( $\%$ area opaque) were derived from Nidek EAS 1000 digitized images. Primary outcome measures were max (OD, OS) for each lens opacity type. Age, demographics, general health, nutritional intake, and solar ocular exposure were measured at baseline. In the crosssectional analyses of baseline data, published in Radiation Research 2009:172:10-20, astronauts who flew in space were matched to comparison subjects (astronauts who had not flown in space, military aircrew, and ground-based controls) using propensity scores based on demographic characteristics and medical history, stratified by gender and smoking (ever/never). We then used various forms of regression analysis (depending upon the statistical properties of each outcome measure) to quantify the effects of space radiation exposure, while controlling for remaining differences in sunlight exposure levels, age at baseline, nutritional intake ( $\beta$-cryptoxanthin and polyunsaturated fats) after matching. For analyses of longitudinal data, we compared cataract progression rates, estimated by median regression, between astronauts who had flown in space, astronauts who had not flown in space, and military aircrew. For astronauts only, we also examined the relationship between cataract progression rates and space radiation exposure history, using a partial correlation analogue of Kendall's Tau to adjust for differences in sunlight exposure, age at baseline, nutritional variables ( $\beta$-cryptoxanthin and polyunsaturated fats), and smoking status (ever/never). Ground-based control subjects (neither astronaut nor military aircrew) were dropped from the longitudinal analyses because they were considered too unlike astronauts in terms of many health/lifestyle characteristics.

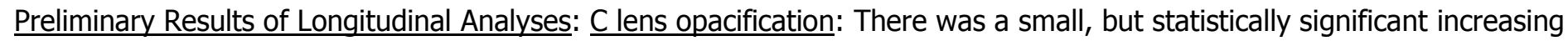
relationship between the rate of cortical opacification and space radiation exposure $(p=0.033$; Kendall's partial correlation $95 \%$ CI $=(0.0064-0.151))$. PSC: There was no statistically significant relationship between the rate of PSC opacification and space radiation exposure. $\underline{\mathrm{N}}$ : There was no statistically significant relationship between slope and space radiation exposure for either measure of nuclear cataract. In similar analyses, there were no statistically significant relationships between rates of change and space radiation exposure for other measures of N, \# Centers of PSC opacification, high- and low-contrast Logmar acuities, or nuclear color.

Preliminary conclusions from the NASCA study: 1 . Exposure to space radiation accelerates cortical opacification. 2. The adverse effect of cortical opacification appears greater relative to the size of opacityat the time of the exposure. 3 . Galactic cosmic ray exposure was linked to increased PSC size and the number of PSC centers. However there were no 
detectable adverse effects of radiation exposure on growth of PSC, change in nuclear color, or change in high-contrast or low-contrast visual acuity. The five-year follow-up period in NASCA is short in the life history of a cataract. Longer follow ups might reveal an association between space radiation exposures, accelerated cortical opacification, and diminished visual acuity.

Support: NASA Grant Number: COOPERATIVE AGREEMENT NUMBER: NAG9-1491

Conflicts of Interest: None 